\title{
Zur Öffentlichkeit der privatvertraglichen Totschlagsühne
}

Tötungsdelikte - Sühne- und Versöhnungsrituale - Steinerne Denkmale

Von BERNHARD Losch

\section{Einführung}

Steinerne Denkmale machen einen großen Anteil unter den Kleindenkmalen aus. Man braucht sich bloß an Grenzsteine, steinerne Bildstöcke oder einfache Denksteine zu erinnern ${ }^{1}$. Dazu gehört auch die Heerschar von alten Steinkreuzen, die über weite Landstriche verbreitet sind ${ }^{2}$. Viele hüllen sich in melancholisch wirkendes Schweigen, verstärkt durch die Einfachheit und Schmucklosigkeit ihrer Gestaltung und natürlich auch dadurch, dass sie von der Last des Alters in den Boden gedrückt sind. Gleichzeitig schwingt ein Knäuel von Assoziationen mit, die von der Kreuzform als religiös überliefertes Todes- und Erlösungssymbol hervorgerufen werden.

In der Tat erinnern die schlichten Steinkreuze größtenteils an plötzliche Todesfälle. Der Brauch, dafür Gedenkzeichen zu setzen, wird nach wie vor geübt, wie zum Beispiel die zahlreichen, meistens hölzernen Kreuze zeigen, die nach tödlichen Verkehrsunfällen an Straßenböschungen aufgestellt werden. Aber unter den tausendfach verbreiteten Steinkreuzen repräsentieren die älteren Exemplare noch einiges an Bedeutung mehr. Sie verkörpern nämlich die Besonderheit, dass sie nach Tötungsdelikten als Teil eines spektakulären öffentlichen Sühne- und Versöhnungsrituals errichtet werden mussten.

\footnotetext{
1 Stellvertretend für viele Dieter Kapff /Reinhard Wolf: Steinkreuze, Grenzsteine, Wegweiser - Kleindenkmale in Baden-Württemberg. 2000. - Günter Meier: Steinkreuze im Kreis Karlsruhe. 1989.

${ }^{2}$ Vgl. Bernhard Losch: Steinkreuze in Südwestdeutschland. 1968. S. 15-17. - Auskunft über neuere Forschungen erteilt die Gesellschaft zur Erhaltung und Erforschung der Kleindenkmale in Baden-Württemberg e.V. (GEEK), c/o Heinz Schmid, Postfach 1127, 72151 Horb (www.kleindenkmale.de).
} 
Deshalb hat sich für die Gruppe der älteren Steinkreuze die Bezeichnung als Sühnekreuze eingebürgert ${ }^{3}$. Aber der Weg dazu war mehr als holprig, denn als sich im 19. Jahrhundert allmählich die Aufmerksamkeit der Landes- und Volkskundler auf die Sachtraditionen und mündlichen Überlieferungen richtete, erschienen die Steinkreuze entweder als normale Gedenkkreuze, wie andere dem „Marterlbrauch“ dienende Gedenkzeichen auch, oder einfach als Teil der verbreiteten Grenz- und sonstigen Hoheitszeichen, die zur Kennzeichnung besonderer Berechtigungen dienten ${ }^{4}$.

Die historische Forschung dagegen stieß immer häufiger auf Dokumente, die erkennen ließen, dass viele der älteren Steinkreuze ihre Aufstellung dem Rechtsbrauch der Totschlagsühne verdanken ${ }^{5}$. Dieser bedeutete, dass nach Tötungsdelikten, wie sie zum Alltag gehörten, anstelle der herkömmlichen Blutrache oder auch der öffentlichen Strafverfolgung ein Vergleich zwischen den Parteien geschlossen wurde, der den Konflikt aus der Welt schaffte. Der Täter verzichtete auf Rechthaberei und weitere Feindseligkeiten. Im Gegenzug verzichteten die Hinterbliebenen auf Rache oder auf Strafverfolgung. Dafür leistete der Täter Schadenersatz.

Einer der Gründe für das Aufleben der Totschlagsühne war, dass bis um 1300 einerseits die Bevölkerung und die soziale Mobilität deutlich zunahmen, andererseits aber die Gerichtsgewalt noch nicht schlagkräftig genug organisiert war, um eine ausreichende Rechtsverfolgung garantieren zu können ${ }^{6}$. Die Lücke, die hinsichtlich der Eindämmung des um sich greifenden Faustrechts in Form von Blutrache und deren organisierter Form ${ }^{7}$, der Fehde, aufklaffte, wurde durch die Aktivierung der althergebrachten Totschlagsühne zu schließen versucht ${ }^{8}$. Dabei entwickelte sich die Totschlagsühne im Mittelalter zu einer Doppelgestalt aus weltlicher Schadenersatz- und Versöhnungsregelung und zugleich aus kirchlich veranlassten Fürbitte- und Bußprozeduren. Einer der kirchlichen Bestandteile, die mit gewisser Regelmäßigkeit in die vertraglichen Abmachungen Eingang fanden, war auch die Verpflichtung, ein Gedenkkreuz für den Getöteten zu errichten?'

${ }^{3}$ Vgl. die Angaben im Internet unter diesem Suchbegriff.

${ }^{4}$ Wie Gerichts-, Markt-, Geleit- oder Meilensteine. - Zur Geschichte der Steinkreuzforschung Losch (wie Anm. 2) S. 65-85.

${ }^{5}$ Ebd. S. 86-93.

${ }^{6}$ Vgl. die Hervorhebung bei Uwe Wesel: Geschichte des Rechts. Von den Frühformen bis zur Gegenwart. ${ }^{3} 2006$. S. 342.

7 So Heinrich Mitteis/Heinz Lieberich: Deutsche Rechtsgeschichte. ${ }^{19} 1992$. S. 40.

${ }^{8}$ Grundsätzlich dazu Andreas Deutsch: Späte Sühne - Zur praktischen und rechtlichen Einordnung der Totschlagsühneverträge in Spätmittelalter und früher Neuzeit. In: ZRG Germ. Abt. 122 (2005) S. 113-149 (mit vielen weiteren Nachweisen).

9 In einem Sühnevertrag vom Ende des 15./Anfang des 16. Jahrhunderts aus der Bodenseegegend wird ausdrücklich zwischen dem Grab- und dem Gedenkkreuz unterschieden, die beide vom Täter geleistet werden mussten. Zit. nach Eytenbenz: Bunte Steine, 4. Hannsen Drayers und seiner Mitverwandten Buß eines Todschlags halber. In: Schriften des Vereins für die Geschichte des Bodensees und seiner Umgebung 2 (1870) S. $226 \mathrm{f}$. 
Typisch wurde dafür das einfache steinerne Kreuz. Die zahlreichen Sühneurkunden, die auch in Baden-Württemberg erhalten geblieben sind ${ }^{10}$, drücken immer wieder aus, dass es sich um einen üblichen Sühnebrauch handle ${ }^{11}$, und häufig wird das Standardmaß angeführt, das sich für Höhe, Breite und Tiefenprofil eingebürgert hatte ${ }^{12}$. Gelegentlich wird auch ausdrücklich die Fertigung aus örtlich anstehendem Werkstein vorgeschrieben ${ }^{13}$. Aus den urkundlichen Nachweisen lässt sich ableiten, dass die einfachen steinernen Kreuze der älteren Zeit in großer Zahl als Sühnekreuze aufgestellt wurden ${ }^{14}$.

Schon die frühesten Beschreibungen von Steinkreuzen stellten den rechtshistorischen Zusammenhang her. Im Gebiet des heutigen Baden-Württembergs ging Benedict Stadelhofer 1787 ausführlich auf die Steinkreuze ein ${ }^{15}$. Er stellte fest, dass sie, wie erhaltene Sühneverträge zeigen, als Teil der Totschlagsühne errichtet werden mussten. Auf Stadelhofer stützte sich im benachbarten Bayern Maurus Feyerabend, der 1814 unter ausdrücklicher Ablehnung der irrigen Deutungen als Markund Leichensteine die alten steinernen Kreuze „als öffentliche Denkzeichen einer

10 Über 50 Vertragstexte und weitere Nachweise; im Einzelnen Bernhard Losch: Sühne und Gedenken. Steinkreuze in Baden-Württemberg. 1981. Kreisübersichten. - Zur Quellenlage vgl. Heiner Lück: Sühne und Strafgerichtsbarkeit im Kursachsen des 15. und 16. Jahrhunderts. In: Hans Schlosser/Dietmar Willoweit (Hg.): Neue Wege strafrechtsgeschichtlicher Forschung. 1999. S. 83-99 (86). - Zu indirekten Nachweisen Hans Jänichen: Schwäbische Totschlagsühnen im 15. und 16. Jahrhundert. In: ZWLG 19 (1960) S. 128-140. - Vgl. eine Abgabenordnung, die sich auf die Herstellung von Sühnekreuzen bezieht, bei M. Johner: Über Kreuzsteine im allgemeinen u. über die Kreuzsteine des Bezirks Ravensburg. In: Landschaft und Kultur im Bezirk Ravensburg 3 (1929) Nr.7, 8 (Nr. 7). Einige neu entdeckte Sühneverträge bei Hans Angele/Johannes Angele: Sühnekreuze im Kreis Biberach, 2012. S. 222-261.

11 Vgl. als eines von vielen Beispielen den Nagolder Sühnevertrag von 1494 mit der Bemerkung: da man gewöhnlich solches zu tun pflegt, zit. nach Anton Nägele: Über Kreuzsteine in Württemberg und ihre Bedeutung. In: Württembergische Jahrbücher für Statistik und Landeskunde 1913. S. 377-426 (403f.).

12 Eine Angabe aus vielen Beispielen lautet: fünff Schuch lanng, dryer brait, und ains Schuchs dick, zit. nach Benedict Stadelhofer: Historia imperialis et exemti Collegii Rothensis in Suevia ex monumentis, domesticis et externis potissimam partem indetitis. Bd.2. 1787. S. 148-162: Dissertatio IV. De saxeis Crucibus in viis publicis, vicinalibus et compitis erectis (S. 148, 154f., 157-162). - Vgl. z.B. auch die in den Sühneverträgen aufgeführten Maße bei Eugen Wiedenmann: Sühnekreuze im Kreis Göppingen. 1978.

${ }_{13}$ So aus Kalktuff laut einem Sühnevertrag von 1520 aus dem Biberacher Kreisgebiet, zit. nach F. Sauter: Todtschläger, wie solche in Schussenried vor der Carolina bestraft worden. In: WVjH 3 (1880) S. 271-273 (272f.). - Ein Kreuz aus Rorschacher Stein wird in einem Sühnevertrag von 1504 aus der Bodenseegegend verlangt. Zit. nach Johner (wie Anm. 10).

${ }_{14}$ Die deshalb auch als Rechtsaltertümer bezeichnet werden und in entsprechenden Forschungen Beachtung finden; vgl. Bernhard Losch: Sühnekreuze und Totschlagsühne in Südwestdeutschland. In: Forschungen zur Rechtsarchäologie und Rechtlichen Volkskunde 17 (1997) S. 83-99. - Siehe ferner die Ausführungen bei Karl Kroeschell: Deutsche Rechtsgeschichte. Bd.1. ${ }^{13} 2008$. S. $144 \mathrm{f}$.

15 Siehe Anm. 12. 
entweder auf dem Platze, wo sie errichtet waren, oder nicht gar ferne davon verübten Mordthat" erklärt, „welche der Mörder auf Begehren der Familie des Erschlagenen errichten musste ...“" ${ }^{\text {"16. }}$

Auf Stadelhofer berief sich 1843 auch August Friedrich von Pauly. Er sah im Sühnevertrag „die geschichtlich bekannte Veranlassung zu den Kreuzen“" ${ }^{17}$. An die älteren Ausführungen von Feyerabend lehnte sich 1861 der Volksforscher Anton Birlinger $a^{18}$. Ebenfalls der rechtshistorischen Deutung folgte 1912 der Steinkreuzforscher Anton Nägele ${ }^{19}$. In erster Linie aber waren es Historiker, die im 19. Jahrhundert auf die Steinkreuze eingingen und ihre Bedeutung als Sühnekreuze herausstellten. Dazu zählt auch Franz Ludwig Baumann, der gegen Ende des 19. Jahrhunderts auf die Steinkreuze und die Totschlagsühne zu sprechen kam ${ }^{20}$.

Als sich von der Mitte des 19. Jahrhunderts an neben der historischen auch die volkskundliche Sach- und Überlieferungsforschung entwickelte, kamen alle möglichen zusätzlichen Deutungsversuche auf ${ }^{21}$, und teilweise kam es zu abenteuerlichen Spekulationen ${ }^{22}$. Nach dem Zweiten Weltkrieg konzentrierte sich die Forschung jedoch wieder auf die wissenschaftlich nachweisbaren Erklärungsweisen. Es zeigte sich, dass es zwar Übergänge zu Steinsetzungen anderer Zweckrichtungen gibt, diese sich aber neben den Sühne- und freiwillig errichteten Gedenkkreuzen mehr oder weniger in Einzelbeispielen verlieren. Dass die Sühnekreuze unter den älteren Steinkreuzen die dominierende Denkmalgruppe bilden, geht aus der enormen Bedeutung der Totschlagsühne hervor.

\section{Öffentliches Drängen auf friedliche Verständigung}

Schon in den meisten germanischen Volksrechten aus dem frühen Mittelalter wird die Sühne näher behandelt und wird auf ihren Abschluss gedrungen ${ }^{23}$. Im

\footnotetext{
16 Maurus Feyerabend: Des ehemaligen Reichsstifts Ottenbeuren Benediktiner Ordens in Schwaben Sämmtliche Jahrbücher in Verbindung mit der allgemeinen Reichs- und der besonderen Geschichte Schwabens diplomatisch, kritisch, und chronologisch in drei Bänden bearbeitet. Von P. Maurus Feyerabend. Benediktiner, und Prior des ehemaligen Reichsstifts. Zweiter Band vom Jahr 1106-1519. 1814. S. 509, $507 \mathrm{f}$.

17 OAB Leutkirch. 1843. S. 103.

18 Anton Birlinger: Aus Schwaben. Sagen, Legenden, Aberglauben, Sitten, Rechtsbräuche, Ortsneckereien, Lieder, Kinderreime. Erster Band. 1874. S.287f., 293. - Vgl. ders.: Volkstümliches aus Schwaben. Erster Band. 1862. S. 169, 171-173.

19 Anton Nägele: Fragen und Ergebnisse der Kreuzsteinforschung. In: Zeitschrift des Vereins für Volkskunde 22 (1912) S. 253-275, 375-398.

${ }^{20}$ Franz Ludwig Baumann: Geschichte des Allgäus von den ältesten Zeiten bis zum Beginne des 19. Jahrhunderts. 2. Bd. Um 1890. S. 326-328.

${ }^{21}$ Siehe Anm. 4.

${ }^{22}$ Etwa zur Deutung als germanische Thing-, Opfer- und Götter- oder als römische Strategie- und Wegzeichen; vgl. Losch (wie Anm. 2) S. 72-78.

${ }^{23}$ Vgl. den Abschnitt „Selbsthilfe, Sühne, Gericht“ bei Kroeschell (wie Anm. 14) S. 35-37
} 
Spätmittelalter erfuhr sie jedoch ihren praktischen Durchbruch auf breiter Linie ${ }^{24}$. Wie alltäglich die Totschlagsühne wurde, zeigt sich nicht nur an der kontinuierlichen Reihe von Sühneverträgen und anderen urkundlichen Nachweisen von Sühneverfahren, sondern auch daran, dass die Verträge selbst auf die Üblichkeit von Sühneabmachungen hinweisen ${ }^{25}$, sowie an zahlreichen historischen Rechtsordnungen, die nicht nur die Sühne regeln, sondern vielfach auch dazu auffordern ${ }^{26}$, und an daran angelehnten Rechtsbüchern ${ }^{27}$. Schließlich wurden Musterbeispiele für Sühneverträge sogar in Formularbücher aufgenommen, die als Vorlage für zeitgemäße Rechtsverfahren dienten ${ }^{28}$.

Die Nachweise machen deutlich, dass der Rechtsbrauch in der Epoche vom 13. bis zum 16. Jahrhundert eine Art Hochkonjunktur erlebte. Mit ihrer Konfliktschlichtungsstrategie war die Totschlagsühne vorzüglich dazu geeignet, die immer wichtiger erscheinende Politik der gesellschaftlichen Friedenswahrung zu unterstützen, die darin ihren Ausdruck fand, dass durch die Verkündung von Gottes-,

sowie den Abschnitt „Quellentexte“ ebd. S.38-45. Ferner Hermann Conrad: Deutsche Rechtsgeschichte I. ${ }^{2} 1962$, S. 168 f.

${ }^{24}$ Grundsätzlich Paul Franenstädt: Blutrache und Todtschlagsühne im Deutschen Mittelalter. 1881. - Ders.: Die Todtschlagsühne des deutschen Mittelalters. In: Sammlung gemeinverständlicher wissenschaftlicher Vorträge NF 1 (1886) S. 373-404. - Zur Sühne fordert auch das Stadtrecht von Isny auf (Ende 14. Jahrhundert). - Dazu und zu weiteren Beispielen Deutsch (wie Anm. 8) S.134-137. - Zur älteren rechtshistorischen Forschung Losch (wie Anm. 2) S. 86-91.

${ }^{25}$ Durch den häufigen Zusatz, der auch auf das Zustandekommen von Sühneverfahren insgesamt zurückschließen lässt, dass Sühneleistungen nach Landesbrauch, Gewohnheit oder Herkommen zu erbringen sind, wie hinsichtlich von Sühnekreuzen (siehe Anm.11) oder von anderen üblichen Sühneleistungen, etwa der Bußprozession des Täters - so beispielsweise in einem Sühnevertrag von 1494 (Tennenbronn, Kreis Rottweil), zit. nach Christian Roder: Der Anteil der Stadt Villingen und des oberen Schwarzwalds an den Ereignissen in Württemberg zur Zeit der Vertreibung Herzog Ulrichs 1519-1522. In: ZGO NF 21 (1906) S. 169-198 (197f.).

26 Z.B. Ordnungen der Stadt Esslingen aus dem 14./15. Jahrhundert, wonach die Rückkehr von Tätern aus der Verbannung vom Abschluss eines Sühnevertrags abhängig gemacht wird, vgl. Karl Pfaff: Geschichte der Reichsstadt Esslingen. 1840. S. 112. - Württembergs ländliche Rechtsquellen. 2. Bd. Bearb. v. Friedrich Wintterlin. 1922. S.145f. - Von „Sühnezwang" im Hinblick auf die üblichen Anweisungen spricht Deutsch (wie Anm. 8) S. 134, 137. -Zum öffentlichen Hinwirken auf die Sühne auch Peter Schuster: Konkurrierende Konfliktlösungsmöglichkeiten. Dynamik und Grenzen des öffentlichen Strafanspruchs im Spätmittelalter. In: Klaus Lüderssen (Hg.): Die Durchsetzung des öffentlichen Strafanspruchs. Systematisierung der Fragestellung. 2002. S. 133-151 (141 f.).

27 So z.B. am Verbrechenstraktat des Albertus Gandinus (13. Jahrhundert) oder am Schwäbisch Haller Rechtsbuch des Conrad Heyden (15. Jahrhundert) zit. nach Deutsch (wie Anm. 8) S. 121-123.

28 So in das Formularbuch von Alexander Hugen: Rethorica und Formulare Teütsch. 1528. ${ }^{3} 1530$, Bl. 62 f. - sowie in die Formularsammlung von Sebastian Meichsner: Formular Hoch oder gemeiner Teutscher Nation. 1567. Zit. nach Kurt Hannemann: Vorläufiges zu Alexander Hugens Alt-Pforzheimer Kanzleibuch von 1528. In: Pforzheimer Geschichtsblätter 1 (1961) S. 29-64 (59-61). 
Königs- und Landfrieden immer wieder zu Ruhe und Ordnung aufgerufen wur$\mathrm{de}^{29}$. Für die Totschlagsühne wirkte sich das neue Bewusstsein zunächst günstig aus. Mit der Zeit wurde es jedoch zum spürbaren Gegenwind, weil es auch zum allmählichen Erstarken einer schlagkräftigen Strafrechtsgewalt beitrug und dafür sogar „die entscheidende Wende ${ }^{\ll 30}$ brachte.

Zunächst aber wurde das Sühneverfahren noch durch die Obrigkeiten gefördert, auch weil diese, worauf noch zurückzukommen ist, eine Gebühr verlangen und sich dadurch eine Einnahmequelle verschaffen konnten. Insbesondere erfuhr es in gleicher Weise, sowohl aus dem Friedenswahrungs- als auch dem finanziellen Grund und außerdem, weil es den kirchlichen Kulten eine wichtige Entfaltungsmöglichkeit und ein besonderes Öffentlichkeitsforum bot, auch lebhafte Förderung durch die mit großem Einfluss agierende Kirche.

\section{Konkurrenz- und Kooperationsverflechtungen mit der Gerichtsbarkeit}

Durch mehrere Jahrhunderte hindurch befand sich die Totschlagsühne in einer Gemengelage mit der Strafgerichtsbarkeit, was von den Sühneverträgen vielfach widergespiegelt wird. Zum einen existierten beide Konfliktlösungssysteme lange Zeit nebeneinander und kamen je nach Aktivitätsentfaltung in der einen oder anderen Richtung zum Zug ${ }^{31}$. So konnten Sühneverhandlungen die Strafverfolgung nicht nur von vornherein verdrängen, sondern auch bewirken, dass eine bereits eingeleitete Strafverfolgung eingestellt werden konnte und sogar schon inhaftierte, angeklagte oder verurteilte Täter durch ein Sühneverfahren wieder frei kommen konnten $^{32}$. Ferner wurden die außergerichtlichen Sühneverfahren in großem Maßstab auch gerichtlich geleitet und in Gerichtsbüchern aufgelistet, wenn auch in der

${ }^{29}$ Vgl. Elmar Wadle: Landfrieden, Strafe, Recht - zwölf Studien zum Mittelalter. 2001.

30 So Kroeschell (wie Anm. 14) S. 211.

31 Zum Nebeneinander von Sühne und Strafrecht Lück (wie Anm. 10) S. 85-89. - Ders.: Zur Entstehung des peinlichen Strafrechts in Kursachsen: Genesis und Alternativen. In: Harriet Rudolph/Helga Schnabel-Schüle (Hg.): Justiz=Justice=Justicia? Rahmenbedingungen von Strafjustiz im frühneuzeitlichen Europa. 2003 (Trierer Historische Forschungen 48). S. 271-286 (279-286). - Ferner Deutsch (wie Anm. 8) S. 131-137. - Grundsätzlich zur Herausbildung des Strafrechts Kroeschell (wie Anm. 14) S. 209-212.

${ }^{32}$ So befreite ein Sühnevertrag von 1434 (Tiengen Kreis Waldshut) den Täter aus dem Gefängnis mit der Folge, dass er die Knechte, die ihn dort bewachten, entlohnen musste. Zit. nach Bader: Urkunden und Regesten aus dem ehemaligen Kletgauer Archive. 2. Abteilung. In: ZGO AF 14 (1862) S. 223-254 (230). - Laut einem Sühnevertrag von 1503 (Wolfach, Ortenaukreis) musste der Täter auch die Kosten tragen, die dadurch entstanden waren, dass er zunächst ins Gefängnis gebracht und vor Gericht gestellt wurde. Zit. nach Franz Disch: Chronik der Stadt Wolfach. 1920. S.360f. - Nach einer Hinrichtung auf Grund eines voreiligen und ungerechten Spruchs des Geschworenengerichts in Tübingen kam es 1323 zu einem Sühnevertrag zwischen der Stadt und den Hinterbliebenen. Zit. nach Jänichen (wie Anm. 10) S. 130. - Dazu auch Deutsch (wie Anm. 8) S. 127. 
Regel nur in Form von Kurzprotokollen, die lediglich das rechtlich relevante Ergebnis in Form der Einigung auf Schadenersatz und Friedenswahrung konstatieren $^{33}$.

Zum andern hatte sich die Sühne auch hinsichtlich von Verfahrensweisen, wie etwa zur Kundmachung der Tat oder zur Anmeldung und Einleitung von Verhandlungen, und außerdem auch inhaltlich, was bestimmte Sanktionen betrifft, wie etwa Verbannungen oder die Sicherung von Annäherungsverboten, Friedlichkeitsgeboten und Friedensgelöbnissen durch Strafen, die gegenüber der Obrigkeit fällig wurden ${ }^{34}$, sowohl an das herkömmliche Prozessrecht angepasst als auch Elemente aus der Entwicklung zur amtlichen Straftatermittlung und zum darauf gestützten Prozess übernommen.

Im Übrigen wurde zwischen Mord- und Totschlagstaten nicht strikt unterschie$\operatorname{den}^{35}$. Zu berücksichtigen ist, dass hinter der Sühne der herkömmliche Gedanke stand, die Geschädigten zu besänftigen und dadurch, wie oben schon hervorgehoben, das Ausufern von weiteren Tötungsdelikten aus Rache zu verhindern, aber nicht, sich gegen Tötungsdelikte grundsätzlich zu verwahren ${ }^{36}$. Eine Rolle dürfte dabei gespielt haben, dass die Sühne, weil sie im örtlich-regionalen Herkommen wurzelte und damit einhergehend soziale Beziehungen, Einflussmöglichkeiten und Interessen von erheblicher Bedeutung waren, weniger an bestimmten Deliktskriterien als an der Konfliktbereinigung unter praktischen Blickpunkten orientiert $\operatorname{war}^{37}$.

Jedoch klingt in den Ordnungen und Vertragsurkunden die Tendenz an, eher den ungeplanten Totschlag als typischen Fall der Sühne zu verstehen. Vermutlich setzte sich die Unterscheidung allmählich durch und kam ein Sühneverfahren eher im Fall eines Totschlags zustande ${ }^{38}$.

Was die örtlich-regionalen und sozialen Einflüsse betrifft, schwanken beispielsweise die Schadenersatzbeträge in den hier zugrunde gelegten Urkunden - abgesehen davon, dass sie je nach den näheren Umständen der Tat und der Zahl und Ersatzerwartung der Betroffenen oder auch nach dem Verhältnis zum Aufwand für

${ }^{33}$ Zahlreiche Beispiele bei Franenstädt: Blutrache (wie Anm.24). - Ausdrücklich auch Lück (wie Anm. 10) S. 70. - Zum Vertragsschluss vor Gericht Deutsch (wie Anm. 8) S. 126.

${ }^{34}$ Vgl. unten Anm. 68-72.

35 Eingehend dazu Deutsch (wie Anm. 8) S.119-125. - Vgl. das angeführte Zitat von Feyerabend (bei Anm. 16).

${ }^{36}$ So zur Charakterisierung des hergebrachten Instruments der Sühne gegenüber dem neueren, prinzipiell präventiven Strafgedanken Schuster (wie Anm. 26) S. $139 \mathrm{f}$.

37 In diesem Sinn auch Deutsch (wie Anm. 8) S. 125.

38 Während die Ordnung der Stadt Esslingen von 1306 noch von Mördern spricht, hat sich im darauf folgenden Jahrhundert in der Ordnung „Vom todschlag“ das Vokabular geändert, siehe Anm. 26. - Vgl. zur Differenzierung bei der Beurteilung der Tötungsdelikte im 16. Jahrhundert: Susanna Pobl: Schuldmindernde Umstände im römischen Recht: Die Verhandlungen des Totschlags im Herzogtum Württemberg im 16. Jahrhundert. In: Rudolph/Schnabel-Schüle (wie Anm. 31) S. 235-256. 
die kirchlichen Leistungen sowie außerdem nach Geldwertschwankungen ohnehin variieren - zwischen 6 und 500 rheinischen Gulden mit einem Schwerpunkt bei Beträgen zwischen 30 und 150 Gulden. Auch die kirchlichen Leistungen bewegen sich lebhaft um einen festen Kern, der bei einer Zahl von 40 bis 60 Seelmessen liegt, wobei zwischen gesungenen und gelesenen Messen, Ämtern und Vigilien unterschieden wird und die Zahl der Priester, welche die Zeremonien durchzuführen hatten, ebenfalls um einen Kern von 30, aber wiederum weit nach oben und unten wechselt, ebenso wie der ähnlich große Umfang der Gefolgschaft des Täters, die teilzunehmen hatte.

Im Einzelnen bestanden wohl ein nicht unbeträchtlicher Spielraum bei der Handhabung der Sühneverfahren und ein gewisses Ausmaß an Willkür. Auch deutet die schon erwähnte, in den Sühneverträgen immer wieder auftauchende Bemerkung, die Ausgleichsleistungen richteten sich nach Landesbrauch, darauf hin, dass es regionale Traditionen der Ausgestaltung gab. Andererseits gleichen die in $\mathrm{Ba}-$ den-Württemberg belegten Sühneverfahren denjenigen in den angrenzenden Ländern und darüber hinaus hinsichtlich sämtlicher Verfahrensbestandteile ziemlich genau und zeigen, dass die örtlich praktizierte Sühne einem allgemein gültigen, standardisierten Schema folgte. Nur die einzelnen Ausgleichsleistungen wurden mehr oder weniger ausführlich behandelt.

Man könnte geneigt sein, dem strafgerichtlichen gegenüber dem Sühneverfahren ein deutlich höheres Maß an Regularität und Objektivität zuzutrauen. Doch ob mit den drastischen Strafen und Verfahrensmethoden und den ebenso örtlich und regional rekrutierten und in das soziale Beziehungsgeflecht eingebundenen Gerichten mehr an Angemessenheit und Gerechtigkeit in die Behandlung der Deliktsfälle gebracht werden konnte, sei dahingestellt. Zu bedenken ist sicher, dass die Sühne zwar als privater Vergleich bezeichnet wird, ihr Zustandekommen und ihre Durchführung aber, wie noch näher auszuführen, in der Hand von Vertretern der öffentlichen Gewalt lag.

Heutzutage gilt die Strafverfolgung als öffentliche Aufgabe, die allein durch die Staatsgewalt ausgeübt werden darf. Die Bereinigung von Straftaten soll jeder privaten Willkür entzogen werden und keinen Konfliktstoff mehr bilden können. Dabei darf der moderne Täter-Opfer-Ausgleich nicht mit der mittelalterlichen Sühne verwechselt werden, die an die Stelle eines Strafverfahrens trat. Der Täter-Opfer-Ausgleich nach $₫ 46$ a des Strafgesetzbuchs stellt lediglich einen Strafmilderungsgrund dar, wonach zugunsten des Täters berücksichtigt werden kann, dass er sich hinsichtlich der Entschädigung des Opfers entgegenkommend verhält.

\section{Verflechtungen mit dem Kirchenregiment}

Die inhaltliche Ausgestaltung der Totschlagsühne hatte sich im späten Mittelalter erheblich gewandelt. Zwar blieb ihr Rechtscharakter als Vergleich zwischen der 
Täter- und der Hinterbliebenenseite mit dem rechtlichen Kern der Schadenersatzleistung unverändert bestehen. Aber aus der weltlichen Rechtsform war das Zwittergebilde eines mit religiösen Praktiken verschlungenen Rechtsinstituts geworden, denn die Täter mussten sich zugleich zu einer reichen Palette religiöser Leistungen verpflichten. Diese dienten sowohl got dem allmechtigen zu lob, und der armen Seel zu trost ${ }^{39}$ als auch der Buße und Absolution des Täters ${ }^{40}$.

Dazu gehörten in erster Linie prunkvolle, in großer Zahl und mit einer Vielzahl von Priestern und Gefolgsmännern abzuhaltende Toten- und Jahrtagsmessen unter Einsatz von Wachskerzen in großer Zahl. Dabei musste die Mannschaft des Täters den Bußcharakter zur Schau stellen, was mit großen theatralischen Gesten zu geschehen hatte, so durch die Einkleidung in Klagegewänder mit übergestülpten Kapuzen $^{41}$ und dadurch, dass brennende oder abgebrochene und angezündete oder auch erloschene oder nach unten gekehrte Kerzen zu tragen waren ${ }^{42}$.

Der zweite wesentliche Teil der religiösen Auflagen bestand aus ebenso feierlich und mit zahlreichem Gefolge zu veranstaltenden Bußprozessionen, an denen der Täter und teilweise auch seine Gefolgschaft in Büßergewand und Büßerhaltung teilzunehmen hatte. In der Regel führten sie um die Kirche herum und zum Grab des Getöteten. Sie waren der Schuldbekenntnis- und Reuedemonstration des Tä-

\footnotetext{
39 Totschlagsühne um 1500 (Ersingen, Enzkreis) zit. nach Hannemann (wie Anm. 28) S. $59 \mathrm{f}$.

${ }^{40}$ Die in den Sühneverträgen häufig als ausführlich zelebrierter Abschluss der Sühneprozession (vgl. Anm. 42) angesprochen wird, so in einem Vertrag von 1582 (Konstanz) zit. nach Wilhelm Fladt: Der Totschlag von Wallhausen und seine Sühne. In: Alemannisches Volk 1938. S. 76. - Teilweise wurde die Absolution auch an auswärtiger Stelle erteilt - so z. B. nach einem Totschlag in einem bischöflichen Dorf durch die österreichische Herrschaft unter der Auflage, einen Sühnevertrag abzuschließen, dessen Vermittlung der Fürstbischof von Basel 1637 zusagte. Zit. nach Bader: Urkunden und Regeste über die ehemalige Hochstift-Baselsche Landvogtei Schliengen (Fortsetzung). In: ZGO AF 17 (1865) S. 356-364 (360f.). - Zusätzlich sollte auch am Wallfahrtsort die Absolution angestrebt werden, so z. B. gemäß einem Sühnevertrag von 1484 (Rot an der Rot, Kreis Biberach) zit. nach Stadelhofer (wie Anm. 12) oder einem Vertrag von 1503 (wie Anm. 32).

${ }^{41}$ Vier der fünf Täter mussten 1447 in grauen Röcken mit vorgestülpten Kappen und nackten Beinen, der Haupttäter mit nacktem Oberkörper und nackten Beinen an der Bußprozession teilnehmen (Michelwinnaden, Kreis Ravensburg) nach Sauter (wie Anm.13). Ähnlich hatte der Täter in schwarzwollenem Kleid, bis auf den Gürtel entblößt und barfuß aufzutreten (1559, Ingoldingen, Kreis Biberach) nach Sauter (wie Anm.13); barfuß mit schwarzer Klagkappe (1514, Rottenburg, Kreis Tübingen) nach Jänichen (wie Anm.10) S. 133-139; schwarz gekleidet im Klagestuhl, anschließend bis auf den Gürtel entblößt (1582, Konstanz) wie Anm. 40.

${ }^{42}$ Vom Täter und seinem Gefolge angezündet mitzuführen (1474, Herrenberg, Kreis Böblingen), zit. nach Abschrift 1980 durch Stadtarchivar Trangott Schmolz. - Ebenso gemäß Sühnevertrag von 1520 (Pforzheim), nach Theodor Schön: Geschichte der Familie Ow. 1910. S.139. - Auf Seiten des Täters mit abgebrochenen und erloschenen Kerzen (1499, Göppingen), nach Wiedenmann (wie Anm. 12) S. 79-82) - oder nach unten gekehrt (1447, Michelwinnaden, Kreis Ravensburg), wie Anm. 41. - Was von den Kerzen übrig blieb, musste jeweils der Kirche oder den Hinterbliebenen gestiftet werden.
} 
ters und seiner anschließenden feierlichen Absolution gewidmet. Er hatte sich teilweise barfuß und bis zum Gürtel entblößt - unter begleitenden Demutsbezeugungen niederzuwerfen und Abbitte zu leisten, bis ihn der Priester wieder aufstehen ließ ${ }^{43}$.

Die Bußprozession fand entweder im Anschluss an die Totenmesse statt oder wurde gesondert davon durchgeführt. Teilweise wurde sie mit der Aufstellung des Gedenkkreuzes verbunden oder führte zu dessen Standort ${ }^{44}$. Was von den Kerzen übrig blieb oder auch zusätzliches Wachs musste der Kirche oder den Verwandten gespendet werden ${ }^{45}$; vielfach mussten anlässlich der Prozessionen auch zusätzlich Kleingeld als Opfer gespendet ${ }^{46}$ oder Brot oder Kleingeld an die Armen verteilt werden ${ }^{47}$.

Außerdem mussten meistens gleich mehrere Wallfahrten an verschiedene Wallfahrtsorte ausgeführt werden. Mit großem Abstand am häufigsten, in der Hälfte der Nachweise, wird der Wallfahrtsort Einsiedeln genannt, es folgt, ebenfalls noch in großer Zahl, zu einem Fünftel, Aachen und seltener Rom ${ }^{48}$. Die Wallfahrtsauflage spiegelt den allgemeinen Wallfahrtskult wider, der zu einer regelrechten Wallfahrtsindustrie ausgebaut war ${ }^{49}$. Dazu gehörte, dass die Verpflichtung zur Wallfahrt durch professionelle Wallfahrer ausgeführt werden durfte, die den

43 Wie, als Beispiel unter vielen, in einem Sühnevertrag von 1447 (wie Anm. 41). Die dort getroffene, häufig gebrauchte Anordnung - kreuzweise auf das Grab zu legen - steht z.B. auch in einem Sühnevertrag von 1496 (Göppingen), nach Wiedenmann (wie Anm.12) S. 7174.

441474 (Herrenberg, Kreis Böblingen) wie Anm. 42. - Ebenso 1494 (Tennenbronn, Kreis Rottweil) wie Anm. 25.

451484 (wie Anm. 40). - 1574 (Hausen ob Urspring, Alb-Donau-Kreis) nach Johner (wie Anm. 10).

461494 (Tennenbronn, Kreis Rottweil) wie Anm.25. - Ebenfalls 1551 (Blumberg, Schwarzwald-Baar-Kreis) nach Franz Ludwig Baumann unter Beihilfe von Georg Tumbült: Mitteilungen aus dem F. Fürstenbergischen Archive. I. Bd. 1894. S. $508 \mathrm{f}$.

471534 (Riedlingen, Kreis Biberach) nach Max Ernst: Alte Steinkreuze in der Umgebung Ulms. In: Ulm und Oberschwaben 29 (1934) S. 1-52 (42). - Ähnlich 1559 (Ingoldingen, Kreis Biberach) wie Anm. 41.

${ }^{48}$ Einsiedeln und Aachen 1434 (Tiengen, Kreis Waldshut) wie Anm. 32. - Z. B. auch 1520 (Pforzheim) wie Anm. 42. - Zusätzlich Wallfahrt nach Rom, 1472 (Esslingen) zit. nach Pfaff (wie Anm. 26). - Wallfahrten nach Rom, Einsiedeln und Inchenhofen 1504 (Bodenseegegend) wie Anm. 13.

49 Vgl. zum Wallfahrtskult Johannes Schmitz: Sühnewallfahrten. Diss. Bonn. 1910. - Lucian Pfleger: Sühnewallfahrten und öffentliche Kirchenbuße im Elsass im späten Mittelalter und in der Neuzeit. In: Archiv für Elsässische Kirchengeschichte 8 (1933) S. 127-162. - Georg Schreiber: Strukturwandel der Wallfahrt. In: Ders. (Hg.): Wallfahrt und Volkstum in Geschichte und Leben. 1934. S.1-183. - Eugen Woblhaupter: Wallfahrt und Recht. Ebd. S.217-242. - Louis Carlen: Wallfahrt und Recht im Abendland. 1987. - Ders.: Straf- und Sühnewallfahrten nach Rom. In: Ders.: Sinnfälliges Recht - Aufsätze zur Rechtsarchäologie und Rechtlichen Volkskunde. 1995. S. 394-416. - Vgl. zum aktuellen Forschungsstand auch die Reihe der Jakobus-Studien, hg. von Klaus Herbers und Robert Plötz, sowie die einschlägigen Forschungen von Jan van Herwaarden. 
Bestätigungsschein zurückbrachten und gegen Unkosten und Provision vermeiden ließen, die Wallfahrten in eigener Person unternehmen zu müssen ${ }^{50}$.

Deutlich wird, dass die Festsetzung der kirchlichen Fürbitte- und Bußleistungen auf einen ausgefeilten Kanon von Kulten zurückgreifen konnte. Die frommen Leistungen mussten nicht erst im Sühneverfahren herausgeformt werden. Vielmehr hatte sich die Tradition aller möglichen religiösen Kulte längst unabhängig davon entwickelt. So war zum Seelenheil von Verstorbenen der Seelgerätskult aufgekommen $^{51}$, an dem verschiedene Kulte beteiligt waren. In die Totschlagsühne fand neben allen möglichen Opfergaben vor allem der Messe-, Prozessions-, Kerzen- oder Wachsspenden ${ }^{52}$ sowie der Wallfahrtskult Eingang.

\section{Verständnisbarrieren}

Doch entgegen der gewaltigen weltlichen und religiösen Bedeutung der Sühne äußern sich Vertreter, die sich der Erforschung volkstümlicher Traditionen widmen, immer noch und immer wieder, wenn auch oft nur andeutungsweise, skeptisch darüber, dass sich die große Gruppe der älteren, einheitlich-schlichten Steinkreuze in beträchtlicher Zahl als Sühnekreuze deuten lässt und dass anders auch gar nicht die Masse und weite Verbreitung dieser Denkmalgruppe mit ihrem unverkennbar einheitlichen Erscheinungsbild erklärt werden kann ${ }^{53}$.

Dementsprechend entbrannte an der Wende zum 20. Jahrhundert ein erbitterter Streit darüber, ob die bekannt gewordenen Sühneurkunden mit ihren Nachweisen über Sühnekreuze überhaupt ernst genommen werden könnten, da sie als absolute Einzelerscheinung und viel zu wenig aussagekräftig missverstanden wurden ${ }^{54}$. Was die Sühnekreuze betrifft, scheint es teilweise auch heute noch an der Anschaulichkeit für die rechtlich veranlasste Ausformung des Steinsetzungskults zu mangeln. Deshalb besteht immer wieder die Neigung, gegenüber der Aufstellung von Steinkreuzen als einfache Totengedenksteine die Gruppe der rechtlich veranlassten Sühnekreuze als bloße Randgruppe zu betrachten.

50 Ausdrücklich festgehalten in einem Sühnevertrag von 1498 (Ulm) zit. nach Horst Wölpert: Die Toten in der Rechtsordnung und dem Brauchtum Schwabens vornehmlich im Mittelalter. Maschinenschriftliche Diss. Tübingen 1963. Anhang.

${ }^{51}$ Franenstädt, Blutrache (wie Anm. 24) S.144-166. - Gertrud Rücklin-Teuscher: Religiöses Volksleben des ausgehenden Mittelalters in den Reichsstädten Hall und Heilbronn. 1933. S. 77-79. - Wölpert (wie Anm. 50) S. 2f., 163 f., 171 (mit weiteren Nachweisen).

52 Vgl. Eugen Woblhaupter: Die Kerze im Recht. 1940. S. 88-94.

${ }^{53}$ Zum zeitgeschichtlichen Hintergrund Losch (wie Anm. 2) S. 70-82.

${ }^{54}$ Grundsätzlich Franz Wilhelm: Alte Stein-Kreuze und Kreuz-Steine im nordwestlichen und westlichen Böhmen. In: Zeitschrift für österreichische Volkskunde 5 (1899) S. 97-113, 202-225. - Ders.: Alte Steinkreuze - Grenzzeichen? - Sühnekreuze! In: Mitteilungen des Vereins für sächsische Volkskunde 4(1906) S. 36-41. 
Umgekehrt hat die rechtshistorische Forschung Probleme damit, den Austauschprozess zwischen rechtlichem Reglement und Alltagswirklichkeit ins Blickfeld zu fassen. Insbesondere scheint die große Zahl von Kirchenkulten, die sich zu nachhaltigen Traditionen ausformten und bei der spät- und nachmittelalterlichen Totschlagsühne eine große Rolle spielten, nur begrenzte Aufmerksamkeit zu finden und weitgehend aus dem Gesichtsfeld, das sich auf den rechtlichen Kern des Tatausgleichs und die Gegenüberstellung von zivilgesellschaftlichem und obrigkeitlichem Rechtsregime konzentriert, ausgeklammert zu werden ${ }^{55}$.

Jedoch zeigt sich gerade am religiösen, mit großem zeremoniellem Aufwand durchgeführten Bestandteil der Sühne sinnfällig, wie stark der Öffentlichkeitseinfluss der Kirche und wie eng ihre Verbundenheit mit der weltlichen Obrigkeit geworden war. Die Kirche hatte sich zu einem wesentlichen Mitgestalter der öffentlichen Ordnung entwickelt und hatte sich ausgedehnte Kompetenzbereiche angeeignet, was zu einem intensiven kirchlichen Hineinregieren ins Alltagsleben führte ${ }^{56}$. Jedenfalls scheinen die kirchlichen Bestandteile einen wesentlichen Beitrag zur sozialen Wirksamkeit und Nachhaltigkeit der alten Konfliktlösungsstrategie - der überkommenen archaischen Privatregulierung - geleistet zu haben.

\section{Öffentlichkeit der privatvertraglichen Sühne}

Eben weil die private Konfliktschlichtung für Gesellschaften typisch ist, die durch keine zentrale Organisation beherrscht werden, sondern aus Volksstämmen und Verwandtschaftsgruppen bestehen ${ }^{57}$, passte sie von der Grundkonzeption her immer weniger in die Zeit, in der sich obrigkeitliche Machtstrukturen mit einer zentralen Herrschaftsorganisation durchsetzten. Aber in der Gegenüberstellung mit dem öffentlichen Strafmonopol wird zwar durchaus zur Kenntnis genommen, dass die Sühne des Spät- und Nachmittelalters in ein öffentlich-rechtliches Ge-

55 Vgl. aber die Hervorhebung der kirchlichen Vertragsbestandteile bei Lück (wie Anm. 10) S. 88 und bei Deutsch (wie Anm. 8) S.116 sowie S. 128 im Rahmen der ausführlichen Besprechung eines Sühnevertrags von 1448 (Schwäbisch Hall).

${ }^{56} \mathrm{Vgl}$. Daniela Müller: Der Einfluss der Kirche. In: Lüderssen (wie Anm. 26) S. 69-93 (72-74). - Friederike Neumann: Öffentliche Sünder in der Kirche des späten Mittelalters. Verfahren, Sanktionen, Rituale. 2008. - Ferner Lotte Kéry: Aspekte des kirchlichen Strafrechts im Liber Extra (1234). In: Schlosser/Willoweit (wie Anm.10) S. 241-297. - Ludger Körntgen: Bußbuch und Bußpraxis in der zweiten Hälfte des 9. Jahrhunderts. In: Wilfried Hartmann unter Mitarbeit von Annette Grabowsky (Hg.): Recht und Gericht in der Kirche und Welt um 900. 2007.

${ }^{57}$ Vgl. Wesel (wie Anm. 6) und S. 31,39-42. - Dazu auch Wolfgang Leiser: Strafrechtspflege in Schwaben vom Mittelalter zur Neuzeit. Ein Überblick. In: ZWLG 45 (1986) S. 9-23 (10f.). 
wand gekleidet wurde ${ }^{58}$, aber vielleicht doch nicht in voller Tragweite, die dazu führte, dass die Sühne gewissermaßen zu einem amtlichen Verfahren mutierte ${ }^{59}$.

Unter diesem Gesichtspunkt lassen die hier herangezogenen Sühneverträge erkennen, dass die Beschreibung der Sühne als Privatregulierung und Sache der privaten Verhandlung zwar auf den Grundcharakter des Rechtsinstituts abstellt, aber seinen praktischen Wandel zu einem öffentlich-rechtlichen Regelungsinstrument übergeht. Zunächst ergibt sich aus den Sühneverträgen, dass die Einleitung und Gestaltung des Verfahrens keine Privatsache war, sondern mindestens mit amtlicher Rückendeckung, in der Regel aber auf herrschaftliche oder amtliche Veranlassung und Vermittlung sowie unter amtlicher Führung vorzunehmen war ${ }^{60}$.

Als ein Fall, bei dem sich die private mit der obrigkeitlichen Handhabung der Sühne überlagerte, wurde 1286 eine Totschlagsschlichtung zwischen Eberhard von Württemberg und Rudolf von Habsburg im Rahmen der Beilegung kriegerischer Auseinandersetzungen vorgenommen ${ }^{61}$. Dagegen tritt 1309 ein Graf mit zwei Schiedsleuten als Vermittler und Verhandlungsführer einer Totschlagsühne auf, die nicht in eigener Angelegenheit, sondern unter Bürgern vereinbart wurde ${ }^{62}$. Das Verfahren war in diesem Fall ausdrücklich und unabhängig vom Stand der Betroffenen in obrigkeitlich-amtliche Hände gelegt.

Die Regel im 15. und 16. Jahrhundert war die Leitung der Sühneverfahren durch Schiedsleute, zu denen gewöhnlich der Schultheiß und der städtische Rat oder wenigstens Ratsmitglieder gehörten, was der Sühne nicht nur einen amtlichen Anstrich, sondern auch eine Art von amtlichem Charakter verlieh. In einem Sühneverfahren von 1450, das den Totschlag unter Bürgern eines Dorfes schlichtete, setzten die drei herrschaftlich zuständigen Grafen den Vergleich zwischen den beiden Tätern und den Hinterbliebenen fest ${ }^{63}$. Ähnlich bildeten 1494 der zustän-

${ }^{58}$ So erörtert Lück (wie Anm.10) S. 92 die praktischen Vorteile und Beweggründe der obrigkeitlichen Durchführung von Sühneverfahren, und Deutsch (wie Anm. 8) S. 134-136 geht ausführlich auf die „Veramtlichung“ des Verfahrens ein. - In diesem Sinn spricht Schuster (wie Anm. 26) S. 137-142 von der Vorrangigkeit der „amtlich kontrollierten Sühne“. Ausführlich zu weltlich-öffentlichen Elementen der Sühne Lück: Zur Entstehung (wie Anm.31).

59 Ausführlich geht jedoch Lück: Zur Entstehung (wie Anm. 31) auf die weltlich-öffentlichen Elemente der Sühne und darauf ein, dass diese insofern in keinem Gegensatz zum Strafverfahren stand, sondern in der langen Übergangszeit der "Zweigleisigkeit" (S. 280) eher die situativen Umstände für die Anwendung der einen oder anderen Konfliktlösungsstrategie verantwortlich waren.

${ }^{60}$ Zur städtisch-herrschaftlichen Zuständigkeit für die Sühne vgl. auch Deutsch (wie Anm. 8) S.134-136.

${ }^{61}$ Vertrag wiedergegeben bei Gustav Wais: Alt-Stuttgart. Die ältesten Bauten, Ansichten und Stadtpläne bis 1800. ${ }^{21954}$. S. 6-11.

${ }^{62}$ Jänichen (wie Anm. 10) S. 129 unter Verweis auf Ludwig Schmid: Geschichte der Pfalzgrafen von Tübingen. 1853. S. 323.

${ }^{63}$ K .Th. Zingeler/G. Buck: Zollerische Schlösser, Burgen und Burgruinen in Schwaben. 1906. S. $118 \mathrm{f}$. 
dige Abt und der zuständige Obervogt ein Schiedsgericht, das die Sühnevereinbarung in Form eines Schiedsspruchs festlegte ${ }^{64}$.

Nach einem Totschlag an einem Amtmann 1511 vermittelte der Herzog von Württemberg das Sühneverfahren ${ }^{65}$. Auf ehemals vorderösterreichischem Gebiet wurden 1514 Regierungskommissionen zur Festsetzung von Sühnevergleichen berufen $^{66}$. Von der Mitte des 16. Jahrhunderts an werden die Schiedsleute häufig ausdrücklich als Schiedsgericht bezeichnet. Der Stockacher Amtmann leitete 1582 einen Sühnevergleich, der vom österreichischen Landesherrn zu billigen war ${ }^{67}$.

Den erhaltenen Urkunden nach, scheint die Abmachung der Sühne zwischen den Parteien, die gewöhnlich in Wirtshäusern stattfand, also zwar auf privater Ebene verhandelt worden zu sein. In Wirklichkeit wurde sie aber durch Schiedsleute eingebracht, erläutert und festgesetzt und war weniger eine private als vielmehr eine offizielle, in amtlich-obrigkeitlicher Regie geführte Angelegenheit.

In die gleiche Richtung weisen auch Vertragsverpflichtungen, die öffentlich-rechtlichen Charakter aufweisen. So werden zusätzlich zu den Leistungen, die der Versöhnung mit den Hinterbliebenen dienten, auch - im Vergleich mit den Schadenersatzbeträgen erhebliche, diese teilweise deutlich übersteigende - Abgaben $^{68}$ oder ausdrücklich als Strafe bezeichnete Zahlungen ${ }^{69}$ an die Obrigkeit festgesetzt. Die Beispiele erinnern nicht nur an die erwähnte Gemengelage mit dem Strafrecht, sondern lassen erkennen, dass die öffentliche Betreuung der Sühne, die aus den Verträgen hervorgeht, als reguläres Amtsgeschäft und die Sühne selbst nicht nur als Vermeidung oder Ersatz, sondern auch als eine Art von regelrechter Strafe verstanden wurde.

Der Öffentlichkeitscharakter kommt außerdem in den schon erwähnten Friedenssicherungssanktionen, wie Aufenthaltsverbote ${ }^{70}$, Kontaktverbote ${ }^{71}$ oder Ver- $^{-}$ bannungen $^{72}$ zum Ausdruck, ferner in Vertrags- und Friedenswahrungsgelöbnissen, die mit öffentlichen Sanktionen für den Fall der Nichteinhaltung gekoppelt

${ }^{64}$ Roder (wie Anm. 25).

${ }^{65}$ Fr. Pressel: Die Unruhen zu Ulm im Jahr 1513. In: ZGO 27 (1875) S. 211-218 (217f.).

66 Jänichen (wie Anm. 10) S. 133-139.

${ }^{67}$ Fladt (wie Anm. 40).

68 Wie etwa in zwei Sühneverträgen von 1514 (wie Anm. 41) und einem Sühnevertrag von 1447 (wie Anm. 41).

69 Z.B. in Verträgen von 1503 (wie Anm. 32), 1551 (wie Anm.46) und 1582 (Geisingen, Kreis Tuttlingen) nach Baumann/Tumbült (wie Anm. 46) II. 1902. S. 388, Nr.528. - In gleicher Höhe wie der Schadenersatz (125 Gulden) wurde 1574 eine Abgabe an die Herrschaft festgesetzt, die zugleich als Gebühr für das Tätigwerden und als zusätzliche Strafe erklärt wurde, zit. nach Eugen Reinert: Von den Kreuzsteinen. In: Tuttlinger Heimatblätter 31 (Juni 1939) S. 1-4 (1-3). - Nachweise zu Gebühr und Strafe auch bei Deutsch (wie Anm. 8) S. 136.

70 Sühnevertrag von 1511 (wie Anm. 65); zwei Sühneverträge von 1514 (wie Anm. 41).

71 Sühnevertrag von 1447 (wie Anm. 41). - Sühnevertrag von 1583 (Riedlingen) nach Jobner (wie Anm. 10).

72 Vergleich von 1549 (Kreis Sigmaringen) zit. nach Baumann/Tumbült (wie Anm. 46) S. 477 f., Nr. 686. - Zur Verbannung als Strafform Leiser (wie Anm. 57) S. 15. 
waren $^{73}$. Auch die vielfach vorgenommene öffentliche Siegelung von Sühneverträgen $^{74}$ lässt diese als mit amtlicher Autorität ausgestattet erscheinen. Die Totschlagsühne entwickelte sich folglich zu einem Doppelgebilde aus privatem Vergleich und amtlichem Management mit amtlicher Verantwortung für ihre Durchführung und ihren Bestand. Damit wurde sie zugleich zu einem Mittel der öffentlichen Strafverfolgung und wirkte wie eine eigenständige, öffentlich-rechtlich organisierte und kontrollierte Sanktion.

Dadurch dass das Verfahren gleichzeitig in die kirchliche Kompetenz für die Lebensordnung eingebunden wurde ${ }^{75}$, verstärkte sich sein öffentlicher Charakter erheblich und wurde die Entwicklung zu einem öffentlichen Sanktionsmechanismus unterstützt. In diesem Zusammenhang scheint es nicht nur so, als ob die kirchliche Ausprägung der Sühne grundsätzlich als allein der religiösen Sphäre zugehörig und für den rechtlichen Vergleich als nebensächlich verstanden würde. Sondern offensichtlich wird zugleich auch das nachdrückliche Bemühen der Kirche, die religiöse Sühne zu einer öffentlichen Angelegenheit zu machen, unterbewertet.

Zum einen wird kaum erwähnt, dass die kirchlichen Sühneprozeduren dem weltlichen Ausgleich einen gleichgewichtigen religiösen an die Seite stellten. So bedeuteten die Leistungen, die für das Seelenheil des Getöteten und seinen Einzug ins Paradies zu erbringen waren, die religiöse Wiedergutmachung und sollten den Hinterbliebenen versichern, dass der Getötete die Erlösung finden konnte. $\mathrm{Zu}-$ gleich wurde der Täter dadurch von seiner Schuld befreit und als derjenige entlastet, der die vorzeitige Abberufung des Getöteten aus dem Leben und die Verkürzung der Möglichkeiten, durch lebenszeitlichen Einsatz das Seelenheil zu erlangen, zu verantworten hatte.

Der Ausgleich, der im Sühnevertrag, wie die Vertragstexte immer wieder hervorheben, dadurch bekräftigt wird, dass die Parteien für alle Zeit versöhnt und die Tat gerichtet und geschlichtet ${ }^{76}$ oder in ewige Vergessenheit versetzt ${ }^{77}$ und dass aller Unwille abgetan $\operatorname{sei}^{78}$, wird durch den kirchlichen Sühneteil auch für die religiöse Glaubens- und Gedankenwelt erzielt. Die geistliche Sühne ergänzt deshalb die weltliche nicht nur, sondern bedeutet ihre komplementäre Vervollständigung. In der weltlich-geistlichen Kombination gibt die Sühne die damalige Auffassung von der Verbundenheit der irdischen mit der überirdischen Existenz wieder. In diesem

${ }^{73}$ Vertrag von 1463 (Weikersheim, Main-Tauber-Kreis) nach Nägele (wie Anm. 11) S. 70$73,402 \mathrm{f}$.

${ }_{74}$ Z.B. in einem Vertrag von 1434 (Tiengen, Kreis Waldshut) wie Anm. 32.

${ }^{75} \mathrm{Vgl}$. auch J.F.H. Abegg: Über den Einfluss der Kirche auf die Sühne bei dem Todtschlag. In: Zeitschrift für Rechtsgeschichte 7 (1869) S.259-279. - Ferner Müller (wie Anm. 56).

76 Sühneverträge von 1484 (wie Anm. 40) und 1494 (wie Anm. 11).

77 Sühnevertrag von 1534 (wie Anm. 47).

78 Sühnevertrag von 1582 (wie Anm. 40). 
Sinn wurde die Sühne nicht nur mit religiöser Weihe, sondern auch mit glaubensgeprägter, psychisch relevanter Nachdrücklichkeit versehen.

Dabei wurde der religiöse Ausgleich, wie geschildert, mit gewaltigem Aufwand zur öffentlichen Anschauung gebracht, und die Veranstaltungen wirkten nicht nur als beeindruckende Zeremonien, sondern erwecken darüber hinaus den Eindruck von Ritualen, mit denen die Vollziehung des geistlich zu Bewirkenden repräsentiert wurde ${ }^{79}$. Sie wurden damit zu einer Inszenierung, die nicht auf das Verhältnis zwischen den Parteien beschränkt blieb, sondern - wie die öffentliche Strafe - eine offizielle Dokumentations-, Beweis- und Durchführungsfunktion erlangte.

Im weltlichen Vertragsbereich wurde der gleiche Effekt durch die Öffentlichkeit und Amtlichkeit der Verhandlung, die erwähnten Vertragseinhaltungs- und Friedenswahrungsgelöbnisse sowie die erwähnte ausdrückliche Hervorhebung der absoluten Schlichtungswirkung erzielt, wobei auch in diesem Fall die zeremonielle Vorgehensweise eine Art von rituellem Charakter annahm und das Verhandlungsergebnis wie eine symbolische Vollziehung zur Darstellung brachte. Die Sühne war demnach, obwohl sie traditionell als private Vereinbarung galt, mit nicht weniger Öffentlichkeitswirksamkeit ausgestattet als die gerichtlich auferlegte und öffentlich vollzogene Strafe.

Aber trotz der Eigenschaft als strafähnliche öffentliche Sanktion gelang es ihr nicht, zu einem Bestandteil der neuzeitlichen Strafverfolgung zu werden. Insofern hatten ihre weltlich-öffentlichen und kirchlich-öffentlichen Ausrüstungen vor dem förmlichen Ausschließlichkeitsanspruch der vordringenden Strafgerichtsbarkeit keinen Bestand. Jedoch dürfte die öffentliche Überformung nicht nur ein wichtiger Grund für den spät- und nachmittelalterlichen Aufschwung der Sühne, sondern auch dafür gewesen sein, dass sie sich gegenüber dem zunehmenden Erstarken des Strafrechts und schließlich sogar teilweise in einer Art illegal gewordener Unterwanderung des öffentlichen Strafanspruchs noch lange Zeit erhalten hat.

\section{Verdrängung durch den öffentlichen Strafanspruch}

Für die Strafgewalt, die immer mehr zum Ausdruck der Souveränität der Obrigkeit und zum Garanten der öffentlichen Sicherheit und Ordnung wurde, musste die privat verankerte Konfliktschlichtung allmählich zu einem Dorn im Auge werden. Mit ihrem Mandat für die Parteien stand sie der Modernisierung und Monopolisierung des Strafrechts, die sich im 16. Jahrhundert mit großen Reformgesetzen ankündigte, nur noch im Weg ${ }^{80}$. Außerdem verlor ihre kirchliche Ausgestaltung

79 Zum rechtsgeschichtlichen Verständnis von Ritualen grundsätzlich Barbara Stollberg-Rilinger: Verfassungsgeschichte als Kulturgeschichte. In: ZRG Germ.Abt. 127 (2010) S. 1-32 (besonders 13-32).

80 Ausführlich dazu und zum allmählichen Verschwinden des Sühnerechts Deutsch (wie Anm. 8) S. 137-147. 
mit der Reformation gewaltig an Boden ${ }^{81}$. Dazu, dass die Zeit nicht nur für die Modernisierung des Strafrechts, sondern ebenso für eine Neubesinnung des pompösen Kirchenwesens reif war, trug im Hinblick auf die Totschlagsühne zweifellos auch bei, dass die kirchlich bestimmten Leistungen immer größeren Umfang eingenommen und das Sühneverfahren stark überlastet hatten.

Die Sühne veränderte sich schließlich von einem viel genutzten Rechtsinstitut zu einer lästigen Tradition, die - im Gegensatz zu ihrer früheren Förderung - immer häufiger bekämpft wurde und gegen die vielfach mit strengen Verboten vorgegangen wurde ${ }^{82}$. Andererseits konnte sie sich in einer Reihe von Gegenden noch bis ins 18. Jahrhundert hinein halten ${ }^{83}$. Auch insofern zeigen sich ihre Wurzeln in einem eher volkstümlichen und lokal-regional gepflegten Herkommen als einer obrigkeitlich-zentralen Organisation ${ }^{84}$, wobei territoriale Macht- und Autoritätsansprüche gerade auch am Festhalten an der Sühne interessiert sein konnten, weil damit Möglichkeiten persönlicher Einflussnahme und Profilierung verbunden waren.

Am dennoch unaufhaltsamen Zurückweichen der Sühne gegenüber dem öffentlichen Strafrecht war eine ganze Reihe von miteinander verschlungenen Gründen beteiligt. Besonders hervorgehoben wird, dass sich gegenüber dem bloßen Rachevermeidungs- der allgemeine Präventionsgedanke immer mehr in den Vordergrund schob. Gegenüber der vorauswirkenden und generellen Tatverhinderung, die sich als Anliegen des Gemeinwohls herausbildete, musste die bloß reaktive, auf eine bestimmte Tat bezogene Konfliktschlichtung als unzulänglich und unzeitgemäß erscheinen $^{85}$.

In diese Richtung könnte sich auch ausgewirkt haben, dass der Ausgleichsgedanke, der die Sühne beherrschte, sich insofern mit dem aufgekommenen generellen

81 In einem Vertrag von 1555 (Schwenningen, Schwarzwald-Baar-Kreis) wurden die kirchlichen Sühneleistungen dem Täter erlassen, da er Protestant war. Zit nach Baumann/ Tumbült (wie Anm. 46) S. 509.

82 Vgl. Deutsch (wie Anm. 8) S.131-149. - In der markgräflichen „Ordnung des Prechthals" von 1575 (Kreis Emmendingen) zit. nach Baumann/Tumbült (wie Anm.46) II. Bd. 1902. S. 230, 238) wird dem Totschläger ausnahmslos Strafe oder Verbannung angedroht, auch für den Fall eines Sühnevertrags.

83 Ausführlich Deutsch (wie Anm. 8) S. 141-147. - In Württemberg wurde die Sühne noch in der neugefassten Landesordnung von 1621 zugelassen. Nach F. Graner: Zur Geschichte der Kriminalrechtspflege in Württemberg. In: WVjH 37 (1931) S. 16-57 (35). - Weitere Hinweise bei Losch (wie Anm. 2) S. 91 f. - Vgl. auch die oben in Anm. 40 erwähnte, 1637 erteilte Zusage zur Vermittlung einer Sühne. - Zum lang dauernden Überleben der Sühne auch Lück (wie Anm. 10) S.86f. - Ders., Zur Entstehung (wie Anm. 31).

84 Vgl. Leiser (wie Anm. 57) S. 13, der auf den spätmittelalterlichen Umschwung von der grundsätzlich gewohnheitsrechtlichen zur gesetzesrechtlichen Rechtspflege hinweist.

85 Vgl. Schuster (wie Anm. 26) S. 145-148. - Auf die Veränderung des Strafzwecks zur Prävention hin geht auch Leiser (wie Anm. 57) S. 16 f. ein. - Beispielsweise wird in der „Ordnung des Prechthals“ von 1575 (wie Anm. 82) die ausnahmslose Androhung von Strafsanktionen damit begründet, dass so vil böser ufsetzliche todschläg begangen werden und durch die Ordnung solche böse übelthaten und todschlag dest ehe vermitten bleiben sollen. 
Präventionszweck nicht vertrug, als er, obwohl die Sühne sich zum strafähnlichen Instrument entwickelt hatte, doch die Strafwürdigkeit der Tat unterlief, weil der Täter weltlich und geistlich völlig entlastet und mit den unbescholtenen Betroffenen und der unbescholtenen Allgemeinheit gleichgestellt und die Tat als ungeschehen behandelt wurde, was dem in den Vordergrund getretenen Unrechts- und Unrechtsbekämpfungsgedanken widersprechen musste.

Demgegenüber wird das lange Zeit auffällige Interesse der Obrigkeit an der Durchführung der Sühne - von der erwähnten Gelegenheit, dabei ein wichtiges Wort mitzureden, abgesehen - gerade auch damit erklärt, dass die Sühne, über den Wiedergutmachungseffekt hinaus, zu einem grundsätzlichen Präventionsinstrument gemacht werden sollte ${ }^{86}$. Dafür war sie letztlich aber doch nicht ausreichend geeignet. Praktisch dürfte auch weniger der aufkommende Präventions- und Gemeinwohlgedanke ausschlaggebend gewesen sein, als vielmehr die Machtinteressen der Territorialherren, denen es darauf ankam, mit Hilfe des Strafrechts ein Gewaltmonopol und ihre Alleinherrschaft durchzusetzen.

Über die egoistische Zielrichtung hinaus trug das Machtstreben auf dem Weg zur zentralistischen Organisation der Gesellschaft schließlich - natürlich vor allem durch seine spätere demokratische Transformation - dazu bei, dass der Ausgleich der partikularen gesellschaftlichen Interessen auf eine zentrale Ebene gehoben und die uneingeschränkte Dominanz der Gruppeninteressen in einen allgemeinen Ausgleich untereinander und mit den Allgemeininteressen verwandelt werden konnte, was auch zugunsten des modernen staatlichen Strafmonopols gilt ${ }^{87}$.

Um weitere Gründe für den Bedeutungsverlust der Sühne anzuführen, dürfte mitgewirkt haben, dass sie trotz ihrer öffentlichen Einkleidung keine mit dem Strafverfahren gleichwertige Hoheitlichkeit erlangte. In diesem Sinn musste die wachsende Gerichtsautorität immer mehr Anstoß daran nehmen, die privaten Parteien mit einer eigenmächtigen Verfahrensrolle ausgestattet zu sehen. Überdies könnte das Bestreben, die Sühne zu einer strafähnlichen Sanktion auszubauen, eine Überforderung des Privatvergleichs dargestellt haben, die sein Zustandekommen unattraktiv werden ließ.

Außerdem dürfte der frühere Grund für die Sühne, ein Gerichtsverfahren vermeiden zu können, angesichts der Verbesserung der Gerichtsorganisation in den Eindruck umgeschlagen sein, der Täter wolle sich ungerechtfertigt der Gerichtsbarkeit entziehen oder solle ihr unrechtmäßig entzogen werden. Dafür dass die Sühne mit der Zeit an Attraktivität verlor, dürften auch wachsende Umgehungsmöglichkeiten durch Flucht oder sich Verbergen und Verweigern beigetragen ha-

${ }^{86}$ Barbara Frenz: Frieden, Gemeinwohl und Gerechtigkeit durch Stadtherr, Rat und Bürger. Strafrechtshistorische Aspekte in deutschen Stadtrechtstexten des 12. und 13. Jahrhunderts. In: Schlosser/ Willoweit (wie Anm.10) S. 111-145.

${ }^{87}$ In diesem Sinn fasst Michael Planen: Ohne Wir kein Ich. Warum wir Egoisten brauchen. 2012. S. 190 die Entwicklung von der Blutrache zum staatlichen Strafmonopol mit den Worten zusammen: „Aus der Spirale der Gewalt wurde damit eine Spirale der Kooperation“. 
ben, sei es, was überhaupt das Zustandekommen eines Verfahrens oder auch die Erfüllung der vertraglich festgelegten Verpflichtungen betrifft ${ }^{88}$.

Ein weiterer Grund für das Zurückweichen der Sühne dürfte sein, dass der Familienverband, auf dem das Schlichtungsverfahren beruhte, allmählich an gesellschaftlicher Bedeutung einbüßte. Außerdem hatte die Notwendigkeit, die Parteien einzubeziehen, eine gewisse Umständlichkeit zur Folge, die - gegenüber der damit verbundenen Möglichkeit, öffentlichen Einfluss wahrzunehmen und Einnahmen zu erzielen - auch zu einer wenig erfreulichen Aufbürdung werden konnte. Die Verträge machen zwar nicht den Eindruck, als wäre die Sühne „zu einem Reichenprivileg degeneriert ${ }^{\text {" } 89}$, sondern beziehen sich auf Täter aus allen sozialen Kreisen, aber, wie schon einmal angeschnitten, liegt die Frage durchaus nahe, für wen und mit welchen Ausgleichserfordernissen ein Sühneverfahren tatsächlich zur Durchführung $\mathrm{kam}^{90}$.

Schließlich dürfte, wie schon mit dem Einfluss der Reformation angedeutet, ein wichtiger Grund im Nachlassen der Überzeugungskraft gelegen haben, die von den kirchlichen Bußprozeduren beansprucht wurde, was vor allem den Gedanken betrifft, sich die Sündenvergebung und das Paradies verschaffen zu können, der besonders in die reformatorische Schusslinie geriet. Letztendlich büßte die Totschlagsühne jedenfalls trotz ihres gebietsweise langen Überdauerns ständig an Effektivität ein ${ }^{91}$, was schließlich zu ihrem völligen Ausklang führte.

\section{Erzählmuster der volkstümlichen Überlieferung}

Im Unterschied zur rechtshistorischen Forschung klammerte die Erforschung der volkstümlichen Überlieferungen, die sich an die Sühnekreuze anlagerten, den Rückgriff auf die historische Herkunft der Denkmale weitgehend aus. Der traditionelle Sagenbestand verbindet die Steinkreuze mit der Erinnerung an Totschlagstaten und Soldatengräber aus alten Kriegen sowie an Unglücksfälle aller Art, wie Blitzschlag und Fuhrwerks- oder Holzfällerunglück. Neben den Hauptmotiven waren Geschichten über alle möglichen anderen Anlässe und über Vorstellungen von der Unheimlichkeit der Standorte verbreitet ${ }^{92}$.

88 Vgl. auch Deutsch (wie Anm. 8) S. 137.

89 Günter Jerouschek: Geburt und Wiedergeburt des peinlichen Strafrechts im Mittelalter. In: Lüderssen (wie Anm. 26) S. 41-52 (52).

90 Die Frage der sozialen Zugehörigkeit der Beteiligten macht Lück (wie Anm. 10) S. $99 \mathrm{zu}$ einem wichtigen Punkt in seiner Auflistung, was bei der Erforschung der Sühne noch der näheren Aufarbeitung bedarf.

91 Die nachlassende Effizienz hebt auch Schuster (wie Anm. 26) S. 148f. hervor.

92 Ausführlich Losch (wie Anm. 2) S. 94-129. - Zum Folgenden Bernhard Losch/Marlies Jörling: Entfremdete Information. Sühne- und Gedenkkreuze in der volkstümlichen Überlieferung. In: Beiträge zur Volkskunde in Baden-Württemberg 4 (1991) S. 273-293. 
Als es im 19. Jahrhundert zur Sammlung von Steinkreuzsagen kam, fiel auf, dass sie, wie sonstige Örtlichkeitssagen auch, Erklärungssagen über die auffälligen Merkmale waren, deren ursprüngliche Herkunft in Vergessenheit geraten war ${ }^{93}$. Die Rede war stets von typischen Beteiligten, wie Bauern oder Handwerksburschen, und typischen Situationen, wie Streit um die Vorfahrt oder aus Eifersucht. Auf diese Weise konnte sich an der Erzählung jedermann beteiligen. Zur Popularität der Geschichten trug bei, dass sie dramatisch ausgeschmückt wurden, zum Beispiel, indem zwei Brüder als Streithähne auftreten, und dass sie mit einleuchtenden Details versehen wurden, indem etwa auf den Denkmalen eingekerbte oder als Relief herausgearbeitete Standes- und Handwerkszeichen ${ }^{94}$, wie Pflugschar oder Zimmermannsbeil, als Tötungswerkzeug fungierten.

Der Zweck der Sagen lag demnach in erster Linie in der Eignung als Gesprächsstoff, aber nicht in der Weitergabe historischer Tatsachen. Das geht auch daraus hervor, dass oft mehrere Erzählweisen als Varianten nebeneinander im Umlauf und die Sagen nicht auf bestimmte Steinkreuze festgelegt waren, sondern sich an anderen Standorten wiederholten, sich vermischten und verzweigten, weshalb sie auch als Wandersagen bezeichnet werden.

Das überwiegende Erzählmotiv, es handle sich um Totschlagkreuze, enthält zugleich auch einen Anklang an den Sühnekreuzbrauch. Konkreter kommt dies beim verbreiteten Sagenmotiv vom gegenseitigen Totschlag zum Ausdruck, das unter den baden-württembergischen Steinkreuzsagen weitaus am häufigsten auftritt. Die Sagenforschung hat den Zusammenhang jedoch nicht begriffen, sondern daran herumgerätselt, was es mit der stereotypen Wiederholung eines so unwahrscheinlichen Falls, dass sich gleich beide Streitenden tödlich verletzen, auf sich haben könnte. Dabei blieben aberwitzige Ideen nicht aus, wie etwa, wenn die Sage auf Mythen vom Zweikampf germanischer Gottheiten zurückgeführt wurde ${ }^{95}$.

In der Tat müsste man am Verstand der Bevölkerung zweifeln, die hartnäckig an der unrealistischen Gegenseitigkeit von Totschlagstaten festhielt, wenn man nicht erkennen würde, dass das Motiv nichts mit der praktischen Wirklichkeit der Tötungstat zu tun hat, sondern sich darin auf abenteuerlich verkürzte, aber vordergründig anschauliche Weise der Sinn der Sühneverfahren erhalten hat. Die Sage lehnt sich zwar sehr wohl an die Tatsache von Totschlagstaten an, aber die Gegenseitigkeit stammt nicht aus dem Tathergang, sondern aus der Erinnerung daran, dass die Tat geschlichtet wurde.

${ }^{93}$ Zum Thema „Rechtssagen“ vgl. die Nachweise bei Losch (wie Anm. 2) S. $106 \mathrm{f}$.

${ }^{94}$ Die ihr Vorbild in Wappendarstellungen finden konnten, vgl. einen Sühnevertrag von 1383 (Ansbach) mit der Anordnung, dass auf dem steinernen Gedenkkreuz gehawen sol werden dez obgenannten Schencken schilt und helm (zit. nach Monumenta Zollerana 5 (1859) S. 133 f.). - Vgl. ferner einen Sühnevertrag von 1520 (Pforzheim) mit der Bestimmung, dass auf dem steinernen Gedenkkreuz sol gehauen sein schüllt und helm Hannsen von Neunegg seligen. Zit. nach Hannemann (wie Anm. 28) S.61.

95 Siehe Anm. 22. 
Dabei konnten die komplizierten Sühneprozeduren nicht zu einem transportablen Sageninhalt komprimiert und ihr religiöser Sinn in keine mündlich weitervermittelbare Erzählform gebracht werden. Aber der weltliche Effekt des Schadensausgleichs und Friedensschlusses konnte mit dem Erzählmuster der Gegenseitigkeit auf den praktischen Punkt gebracht und zu einer anschaulichen Formel geprägt werden. Zu denken gibt natürlich, dass die Versöhnung verkannt und der Ausgleich auf die brachiale Beseitigung des Konflikts schon im Tathergang vorverlegt wurde. Vielleicht schwingt dabei eine Anlehnung an das ältere Prinzip der Blutrache und Tatvergeltung mit.

Wie die übrig gebliebenen Denkmale, die aus der ehemaligen Verbundenheit von weltlicher Konfliktschlichtung und religiöser Sündenerlösung herrühren, inzwischen zu Überbleibseln einer überholten Epoche geworden sind, so haben sich auch die alten Sagen weitgehend verflüchtigt und in die Sagenbücher zurückgezogen. Doch während die Denkmale neue Funktionen im Rahmen der Ortsbild- und Landschaftspflege gewinnen, werden die Sagen durch die historische Kenntnis von der wahren Herkunft der Denksteine, über die sie fabulieren, ersetzt, und man wird ihrem Verlust nicht nachtrauern können. 